\title{
Short term Load Forecasting Considering Demand Response under virtual power plant mode
}

\author{
Zhendong Du ${ }^{1}$, Di Wu ${ }^{1}$, Hua Bai ${ }^{1}$, Zhengyong Wang ${ }^{1}$, Yizong Guo ${ }^{2 *}$ and Chuangxin Guo ${ }^{2}$ \\ ${ }^{1}$ Zhejiang Huayun Electric Power Engineering Design \& Consultation Co., Ltd., Hangzhou 310014, China \\ ${ }^{2}$ College of Electrical Engineering, Zhejiang University, Hangzhou 310027, China
}

\begin{abstract}
In order to better manage demand response resources of user side and reduce short-term load forecasting error, a short-term load forecasting method considering demand response in virtual power plant mode is proposed. Firstly, the demand response mechanism of the virtual power plant is analyzed. Taking the maximum profit of the virtual power plant as the goal, considering the user's energy consumption habits, self built photovoltaic, energy storage behavior and thermal electric coupling, the optimization model is established for each type of demand response resources. The CPLEX solver is called to solve the mixed integer linear programming problem after the model transformation, and the sub signals of each resource participating in the demand response are obtained. Then, based on this model, a long-term and short-term memory network model considering demand response signals is established to predict load power iteratively. At the same time, the long-term and short-term memory network model considering the demand response signals effectively makes up for the shortcomings of the traditional forecasting model without considering the demand response, and is more accurate in predicting the future trend of load change.
\end{abstract}

\section{Introduction}

Demand response technology can fully tap the user side resources, guide users to actively participate in the operation and regulation of the power market, realize the optimal allocation of a variety of resources, and also play a greater role in promoting the peak load and valley filling of the power system. It is one of the key technologies to promote the construction of ubiquitous power Internet of things [1-2]. The research on demand side is no longer only oriented to the demand response of power load, but includes the integrated demand response (DR) of various load forms, energy storage and distributed generation [3]. The demand response resources are more decentralized, the load elasticity level often can not meet the demand response requirements.

Many scholars at home and abroad have done a lot of research on short-term load forecasting methods, but most of them are limited to a single power system, without considering the impact of demand response of virtual power plant mode, and the forecasting results can not well serve the follow-up research of virtual power plant mode [4-6]. Reference [7] establishes the mechanism of fuzzy demand response based on logistic function; reference [8] studies the short-term load forecasting method considering demand response in active distribution network. Reference [9-10] models and analyzes the demand response considering virtual power plant mode, and introduces it into RBF neural network model for short-term load forecasting.
In order to better predict the load change under the virtual power plant mode, this paper first analyzes the demand response mechanism of the virtual power plant. Then, a load forecasting model of long short term memory network (LSTM) is proposed, which does not include DR signal and DR signal, and an example is given to analyze the actual load data of a certain area. CPLEX solver has been used to solve DR sub signal, and take it as a part of LSTM input layer. The example results verify the feasibility of virtual power plant mode and the load forecasting model with DR signal has better forecasting performance.

\section{DR signal model based on virtual power plant mode}

In VPP mode, the load classification standard and the load elasticity of demand response resources will affect the signal results. Therefore, it is necessary to establish the demand response mechanism of virtual power plant, as shown in Figure 1.

Among them, virtual power plant can be an intermediary company for the purpose of profit. In the spot market, it signs bilateral contracts with independent system operators to meet the demand of peak load cutting and valley filling of power system, and obtains the load compensation income paid by independent system operators at the grid real-time price. Users give part of the decision-making power of their own power consumption plan to the virtual power plant to implement, so as to provide guarantee for the virtual power plant to

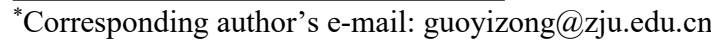


respond to the needs of the superior independent system operators, and accordingly they will get economic rewards paid by the virtual power plant[11-12].

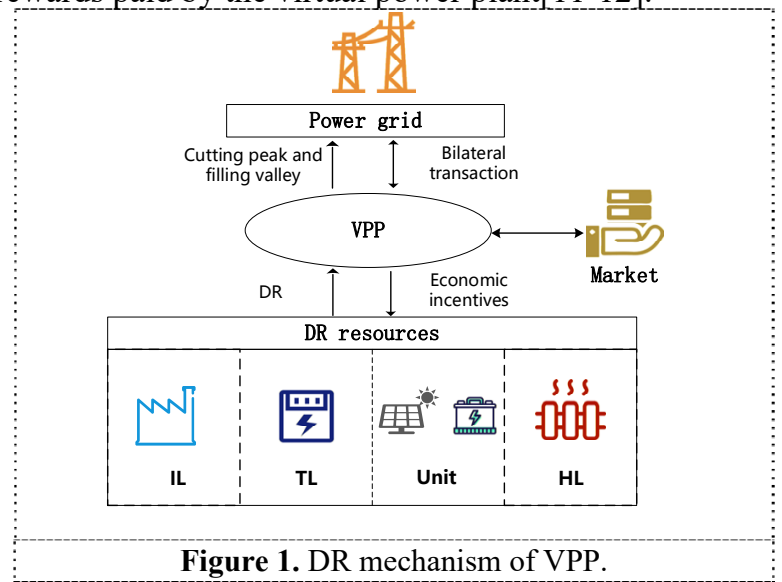

\subsection{DR sub signal with interruptible electrical load}

Under the environment of real-time electricity price, virtual power plant optimizes the power consumption plan of the interruptible load managed in the region, and takes the profit maximization of virtual power plant as the optimization goal. The DR sub signal of IL was obtained by the following optimization model.

$$
\begin{gathered}
V_{\max }^{I L}=\sum_{t \in T}\left(\rho_{t} I L_{t}-\sum_{i \in N} c_{i, t} P_{i, t}^{I L} x_{i, t}\right) \\
I L_{t}=\sum_{i \in N} P_{i, t}^{I L} x_{i, t}
\end{gathered}
$$

The electricity price contract signed by the virtual power plant and the IL user contains the demand response rules, which are reflected in the optimization problem as constraints. Among them, the amount of load interruption at any time and the total power consumption reduction in a day for each user shall not exceed the agreed limit.

$$
\begin{gathered}
0 \leq P_{i, t}^{I L} x_{i, t} \leq P_{\max } \forall i \in N, \forall t \in T \\
0 \leq \sum_{t \in T} P_{i, t}^{I L} x_{i, t} t \leq E_{\max } \forall i \in N
\end{gathered}
$$

The duration of demand response needs to be limited. At the same time, from the psychological point of view of users' load reduction, users do not want to reduce the load many times, but expect at most one continuous reduction in a day.

$$
\begin{gathered}
T_{\min } \leq \sum_{t \in T} x_{i, t} \leq T_{\max } \forall i \in N \\
0 \leq \sum_{t \in T}\left|x_{i, t+1}-x_{i, t}\right| \leq 2 \forall i \in N
\end{gathered}
$$

\subsection{DR sub signal of transferable electric load}

Through the guidance of real-time electricity price, transferable electricity load can actively guide users to transfer part of electricity load from peak electricity price period to low electricity price period, so as to ensure that the total load in a day remains unchanged. In VPP mode, the demand response scheduling mode for TL users is similar to that for IL users, and the optimized objective function is consistent, so it will not be repeated here. However, the overall constraints are slightly different, which are listed as follows:

$$
\begin{gathered}
\left|P_{i, t}^{T L} x_{i, t}\right| \leq P_{\max } \quad \forall i \in N, \forall t \in T \\
\sum_{t \in T}\left|P_{i, t}^{T L} x_{i, t}\right| t \leq E_{\max } \forall i \in N \\
\sum_{t \in T} P_{i, t}^{T L}=0 \quad \forall i \in N \\
T_{\min } \leq \sum_{t \in T} x_{i, t} \leq T_{\max } \forall i \in N \\
0 \leq \sum_{t \in T}\left|x_{i, t+1}-x_{i, t}\right| \leq 4 \forall i \in N
\end{gathered}
$$

\subsection{DR sub signal of photovoltaic and energy storage unit}

Combined with the above energy hub structure, the charging and discharging behaviors of the users controlled by the virtual power plant are analyzed as follows: when the photovoltaic output supplied to the users of the optical storage unit $P_{i, t}^{P V}$ is greater than the fixed load $P_{i, t}^{F L}$ or the time price is the low price, the energy storage charges; when all the photovoltaic output $P_{i, t}^{P V}$ is less than the fixed load $P_{i, t}^{F L}$ and the time price is the peak price, the energy storage discharges.

The mathematical model of user's charge and discharge behavior in each period is as follows.

$$
\begin{aligned}
& \left\{\begin{array}{l}
P_{i, t}^{C}=\min \left\{P_{i, \max }, \frac{E_{i, \max }-E_{i, t-1}}{\eta_{i}^{C} \Delta t}\right\}, \rho_{t} \in \rho_{g} \\
P_{i, t}^{F}=0
\end{array}\right. \\
& \left\{\begin{array}{l}
P_{i, t}^{C}=\min \left\{P_{i, \max }, \frac{E_{i, \max }-E_{i, t-1}}{\eta_{i}^{C} \Delta t}, P_{i, t}^{B}\right\} \\
P_{i, t}^{F}=\min \left\{P_{i, \max }, \frac{\eta_{i}^{F}\left(E_{i, t-1}-E_{i, \min }\right)}{\Delta t}, P_{i, t}^{G}\right\}
\end{array}, \rho_{t} \in \rho_{f}\right.
\end{aligned}
$$

In addition to the constraints of equations (10) - (13), the following constraints are listed according to the physical characteristics of the energy storage equipment:

$$
\begin{gathered}
0 \leq P_{i, t}^{C}, P_{i, t}^{F} \leq P_{i, \max } \\
E_{i, t}=(1-\delta) E_{i, t-1}+\Delta t\left(\eta^{C} P_{i, t}^{C}-\frac{P_{i, t}^{F}}{\eta^{F}}\right)
\end{gathered}
$$

\subsection{DR sub signal of heating load}

In the indoor comfortable temperature range, small changes in temperature will not affect the comfort of human sensory. Therefore, virtual power plant can establish demand response contract with heating load on the premise of ensuring customer satisfaction. Firstly, it is necessary to establish the DR sub signal model of heating load. The indoor temperature of buildings is the 
control quantity, so it is necessary to establish the relationship between heat load and indoor temperature.

$$
\begin{gathered}
T_{i n, t+1}=T_{i n, t} e^{-\Delta t / \tau}+\left(R Q_{t}+T_{\text {out }, t}\right)\left(1-e^{-\Delta t / \tau}\right) \\
\tau=R C
\end{gathered}
$$

\section{Short term load forecasting}

\subsection{LSTM model}

Long term and short term memory network is a variant of recurrent neural network. LSTM is similar to the standard recurrent neural network, but it also has a chain structure, but it is different: unlike the standard recurrent neural network, there is only one neural network layer in the repetitive module, while LSTM is four network layers, which interact in a special form.

The core of the long-term and short-term memory network is the introduction of a new neuron state, which transmits linear circular information and outputs information to the hidden state, as shown in Figure 2. The state of a neuron is like a conveyor belt that runs through the chain.

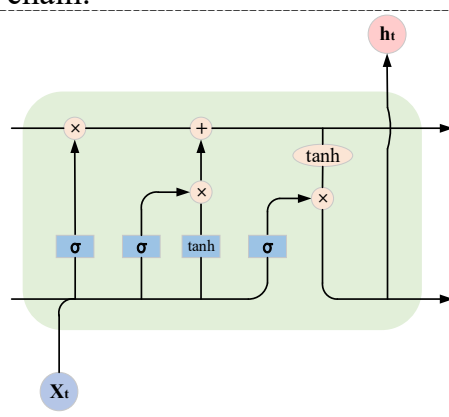

Figure 2. Long Short Term Memory Model.

LSTM also chooses to keep or forget information through three control gate units (input gate, forgetting gate and output gate). The input gate (18) controls how much input information at the current time needs to be retained; the forgetting gate (19) controls how much information at the previous time needs to be discarded; and the output gate (20) controls how much information at the current time needs to be output to the hidden state. The formula of each time (step) state update of long-term and short-term memory network is as follows.

$$
\begin{gathered}
h_{t}=g_{o}^{(b)} f_{h}\left(C_{t}\right) \\
C_{t}=g_{f}^{(t)} C_{t-1}+g_{i}^{(t)} f_{s}\left(w h_{t-1}+u X_{t}+b\right) \\
g_{i}^{(t)}=\operatorname{sigmoid}\left(w_{i} h_{t-1}+u_{i} X_{t}+b_{i}\right) \\
g_{f}^{(t)}=\operatorname{sigmoid}\left(w_{f} h_{t-1}+u_{f} X_{t}+b_{f}\right) \\
g_{o}^{(t)}=\operatorname{sigmoid}\left(w_{o_{i-1}}+u_{o} X_{t}+b_{o}\right)
\end{gathered}
$$

In order to solve the problem of gradient dispersion or gradient explosion when the sequence information is too long, the selective forgetting or preserving state information of input gate, forgetting gate and output gate unit is used.

\subsection{Short term load forecasting based on LSTM}

The short-term load forecasting model in the previous section does not consider the demand response, and only relies on a single input of historical load value to forecast the load value with demand response. If the regional demand response is implemented, the impact of demand response can be considered into load forecasting. The influence of demand response is converted into demand response signal, which is input into the model network together with the historical load value.

For the preprocessing process of input data, it is not only necessary to normalize the load data, but also to normalize the DR signal obtained by CPLEX solver, so as to eliminate the difference of dimension and numerical value between quantities. The iterative load forecasting method is also used, which is the same as that without considering DR signal.

\section{Example analysis}

In order to compare the performance differences of shortterm load forecasting models considering demand response signals or not, this example uses the actual data of a regional power grid and the contract of virtual power plant to calculate the above DR resource sub signals, introduces the total DR signal into LSTM model, and compares the LSTM short-term load forecasting model without considering demand response signal and LSTM short-term load forecasting model considering DR signal in virtual power plant mode The prediction performance of the prediction model.

\subsection{Solution of DR total signal}

This example selects the contract data of virtual power plants and users in PJM market of the United States.

By solving the above optimization model of DR resources, the revenue of each DR sub signal and each DR resource of virtual power plant can be obtained. The latter is shown in Table 1. The results show that the virtual power plant can obtain certain benefits by using the price difference between the real-time electricity price and the contract electricity price and reasonably arranging the response plan of each DR resource.

Table 1 Optimal revenue of DR resources of VPP

\begin{tabular}{ccccc}
\hline & IL & TL & Unit & HL \\
\hline DR profit/\$ & 357.63 & 298.32 & 319.42 & 127.11 \\
\hline
\end{tabular}

Summarize the optimized DR sub signals of each resource to form the total DR signal of LSTM model input layer. In this example, the positive value is the load power cut, and the negative value is the load power transferred into the period or the energy storage charging power.

According to the DR signal analysis, the virtual power plant will arrange the DR resources to reduce the load during the peak period of electricity price, and arrange the load transfer and energy storage charging 
during the low period of electricity price, which also meets the requirements of peak load cutting and valley filling.

\subsection{Analysis of prediction results}

As shown in Figure 3, the historical load data of a certain day in the test set is selected, and the red curve DRawn is the actual load data. The green curve and the blue curve represent the load forecasting curve without DR signal and the load forecasting curve with DR signal respectively.

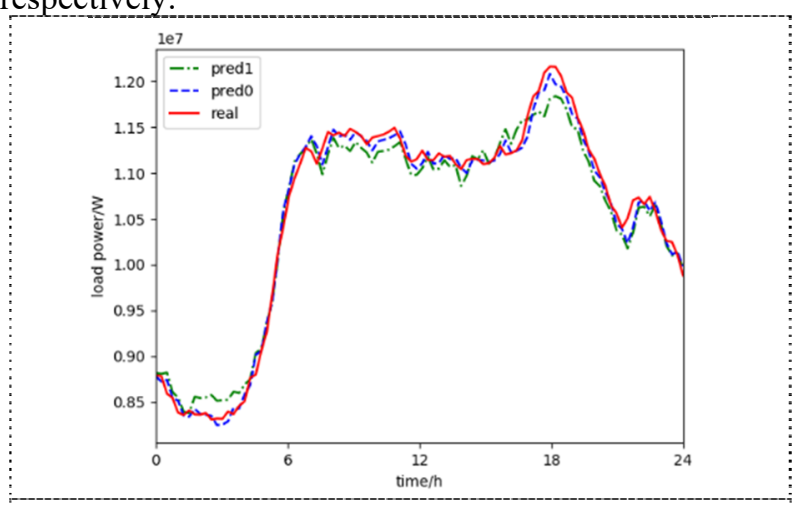

Figure 3. The load prediction comparison of account for DR signals.

The prediction error without DR signal is larger than that with DR signal. Taking the daily load actual value and predicted value of the curve in Figure 4 as an example, the maximum relative error before and after DR signal is calculated to be $1.43 \%$ and $0.85 \%$ respectively. This result fully shows that the load forecasting model considering DR signal has better performance.

Generally speaking, although the model considering DR signal increases the dimension of input quantity, which makes the model more complex in structure and training, after the introduction of virtual power plant, the load forecasting considers the demand response signal, which can make a better judgment on the load change trend.

\section{Conclusion}

Combined with the demand of virtual power plant for load forecasting, a short-term load forecasting method based on demand response signal is proposed by using long-term and short-term memory neural network. The main research results are as follows

1) The demand response mechanism of virtual power plant is analyzed. The optimization models of four DR resources are established. The results show that the DR sub signals of virtual power plant are in favour of enhancing the accuray of load forcast.

2) In the aspect of forecasting method, based on the LSTM load forecasting model with single load input, the LSTM load forecasting model considering DR signal is proposed.

3) Taking the historical load of a region to implement demand response as an example, the maximum relative error of prediction before and after DR signal is $1.43 \%$ and $0.85 \%$, respectively. The error is reduced, the prediction effect is better, and the load fluctuation can be better predicted.

The short-term load forecasting considering DR signal in the virtual power plant mode studied in this paper has a certain engineering practical value, which is conducive to the follow-up virtual power plant to more accurately control the demand response resources, and plays a promoting role in the virtual power plant planning, scheduling, electricity price and other aspects.

\section{Acknowledgments}

This work was supported by the Technology Project of Zhejiang Huayun Electric Power Engineering Design \& Consultation Co., Ltd.(2019C1D09P01) and the National Key Research and Development Program of China under Grant 2016YFB0900100.

\section{References}

1. Yang Ting, Zhai Feng, Zhao Yingjie, et al. Explanation and prospect of ubiquitous electric power Internet of things[J]. Automation of Electric Power Systems, 2019, 43(13): 9-20+53.

2. WANG Beibei. Research on consumers' response characteristics under ability smart grid: a literatures survey[J]. Proceedings of the CSEE, 2014, 34(22): 3654-3663.

3. Bahrami S, Sheikhi A. From Demand Response in Smart Grid Toward Integrated Demand Response in Smart Energy Hub[J]. IEEE Transactions on Smart Grid, 2015: 1-1.

4. Liao Nihuan, $\mathrm{Hu}$ Zhihong, Ma Yingying, et al. Review of the short-term load forecasting methods of electric power system[J]. Power System Protection and Control, 2011, 39(01): 147-152.

5. Xia Bo, Yang Chao, Li Chong. Review of the shortterm load forecasting methods of electric power system [J]. Power Systems and Big Data, 2018, 21(07): 22-28.

6. Dudek G. Artificial Immune System For Short-Term Electric Load Forecasting[J]. IEEE Transactions on Evolutionary Computation, 2017, PP(99): 1-1.

7. Zhang Zhisheng, Yu Daolin. RBF-NN based short term load forecasting model considering comprehensive factors affecting demand response $[\mathrm{J}]$. Proceedings of the CSEE, 2018, 38(06): 1631$1638+1899$.

8. Su Xiaolin, Liu Xiaojie, Yan Xiaoxia, et al. Shortterm load forecasting of active distribution network based on demand response[J]. Automation of Electric Power Systems, 2018, 42(10): 60-66+134.

9. Chen Lina, Zhang Zhisheng, Yu Daolin. Short-term load forecasting model of power system based on generalized demand side resources aggregation[J]. Power System Protection and Control, 2018, 46(15): 45-51. 
10. Peng Wen, Wang Jinrui, Yin Shanqing. Short-term load forecasting model based on Attention-LSTM in electricity market $[\mathrm{J}]$. Power System Technology, 2019, 43(05): 1745-1751.

11. GUO Yizong, WANG Chutong, SHI Yunhui, et al. Comprehensive optimization configuration of electric and thermal cloud energy storage in regional integrated energy system $[\mathrm{J}]$. Power System Technology, 2020, 44(5):1611-1621.

12. GUO Yizong, FENG Bin, YUE Boxiong, et al. Ultra-short-term Load Forecasting Considering Demand Response in Load Aggregator Mode[J]. Automation of Electric Power Systems, 2021, 45(1):79-87. 